\title{
Corrigendum to: Variable data measurement systems analysis: advances in gage bias and linearity referencing and acceptability
}

Corrigendum for: Int. J. Metrol. Qual. Eng. 11, 16 (2020) - https://doi.org/10.1051/ ijmqe/2020010

Mahjoub Abdelgadir ${ }^{*}$, Chris Gerling ${ }^{1}$, and Joel Dobson

Texas Instruments Inc. Quality Standards and Statistical Services, Dallas, TX 75243, USA

Received: 12 September 2020 / Accepted: 23 October 2020

The original article published with below error on December 11th, 2020 was reported by the authors.

Equation (3) should read:

$$
\bar{x}-\mu=\mathrm{T}_{\text {crit }} \cdot(\mathrm{S} / \sqrt{\mathrm{m}})
$$

instead of:

$$
\bar{x}-\mu=\operatorname{Tcrit} /(\mathrm{S} / \sqrt{\mathrm{m}}) \text {. }
$$

\footnotetext{
* Corresponding author: m.a.gadir53@gmail.com

${ }^{1}$ Retired in 2017. Consultant.
} 